\title{
High-Resolution Inline Density Measurements: Insight on Multiphase Flow and Transport Phenomena in Porous Media
}

\author{
Jelayne Falat $^{1}$, Adam Fehr $^{1}$, Ali Telmadarreie ${ }^{1}$, and Steven Bryant ${ }^{1,2}$ \\ ${ }^{1}$ Univeristy of Calgary, Chemical and Petroleum Engineering Department, 2500 University Dr. NW T2N 1N4 \\ ${ }^{2}$ Canada Excellence Research Chair in Materials Engineering for Unconventional Oil Reservoirs
}

\begin{abstract}
Understanding fluid flow in porous media is essential with complex and multiphase fluid flow. We demonstrate that high-resolution in-line density measurements are a valuable tool in this regard. An in-line densitometer is used in fluid flow in porous media applications to quantify fluid production and obtain quantitative and qualitative information such as breakthrough times, emulsion/foam generation, and steam condensation.
\end{abstract}

In order to determine the potential applications for in-line densitometry for fluid flow in porous media, a series of sand pack floods were performed with a densitometer placed at the outlet of a sand pack. All fluids passed through the measurement cell at experiential temperatures and pressures. An algorithm was developed and applied to the density data to provide a quantitative determination of oil and water production. The second series of tests were performed at high temperature and pressure, with a densitometer placed at the inlet and outlet of a sand pack, for steam applications. In both series of experiments, data acquisition was collected at 1 hertz and the analyzed density data was compared to results from the conventional effluent analysis, including Dean-Stark, toluene separations, magnetic susceptibility measurement, and flash calculations where applicable.

The high-resolution monitoring of effluent from a flow experiment through porous media in a system with two phases of known densities enables two-phase production to be accurately quantified in the case of both light and heavy oil. The frequency of measurements results in a high-resolution history of breakthrough times and fluid behavior. In the case of monitoring steam injection processes, reliable laboratory tests show that in-line density measurements enable the determination of steam quality at the inlet and outlet of a sand pack and qualitative determination of steam condensation monitoring

The use of in-line densitometry provides insight on the monitoring of complex fluid flow in porous media, which typical bulk effluent analysis is not able to do. The ability to measure produced fluids at high resolution and extreme temperatures reduces mass balance error associated with the effluent collection and broadens our understanding of complex fluid flow in porous media.

\section{Introduction}

Traditional core flood systems utilize two/three-phase separators or fraction collectors downstream of the core to collect effluent. While phase separators prove reliable in conventional applications, they are limited in their ability to handle highly viscous samples and complex fluids like emulsions and nanofluids. Fraction collectors may cause an error in mass balance, due to misalignment of tubes, rate of sample entering tubes, movement of the collector's arm between tubes, etc. Additionally, fraction collectors result in low temporal resolution, as all effluent is mixed in the tube, resulting in an average composition over a given tube volume. Once the effluent is collected, analyzing it can be both time-consuming and challenging [1]. The dean-stark analysis is accurate; however, the temporal resolution is very low and, in many cases, only a single end-point oil production value is obtained. Methods such as NMR, solvent extraction, Karl-Fischer, and the like are costly, time-consuming, and limited to the resolution of the effluent collection method. In-line densitometry enables real-time quantification of produced water and oil and gives a valuable qualitative understanding of phase behavior and fluid flow in porous media.

A high-temperature and high-pressure density meter can be integrated into a core flood set-up to measure effluent density and distinguish between crude oil and injection fluid. The method discussed involves weighing the produced oil to determine the weight of the residual oil remaining. The density measurements are used along with effluent analysis to facilitate laboratory studies of enhanced oil recovery.

Olsen et al. (2017) described a method for using a densitometer for quantifying oil production in two- 
phase core flood experiments and looked at the understanding obtained by in-line density data for dynamic events such as water breakthrough, gas breakthrough, and connate water production. They conducted immiscible displacements at constant temperature and pressure conditions using North Sea light crude oil and were able to determine that the densitometer produced comparable data to that from an acoustic separator [2].

In this paper, we look at the potential applications and the challenges of utilizing in-line densitometry in core flooding. We demonstrate the benefit of using a high temporal resolution effluent analysis technique as a tool for understanding phase behavior and fluid flow in porous media. We look at the challenges of quantifying heavy oil and assess the use of in-line densitometry in emulsion, foam, and nanofluid core flood experiments. Additionally, we look at the applications of in-line densitometry for steam flooding and the potential understanding gained from being able to determine dynamic water saturation histories during hot gas flooding, condensation monitoring for steam flooding, and real-time volumetric steam quality data. Conventionally, acquiring such information is challenging and may require complex dynamic imaging techniques (i.e. CT, MRI) which are costly. This paper introduces easy and continuous methods using high-resolution densitometry for measuring and analyzing the data that cannot be obtained with conventional core flood configurations.

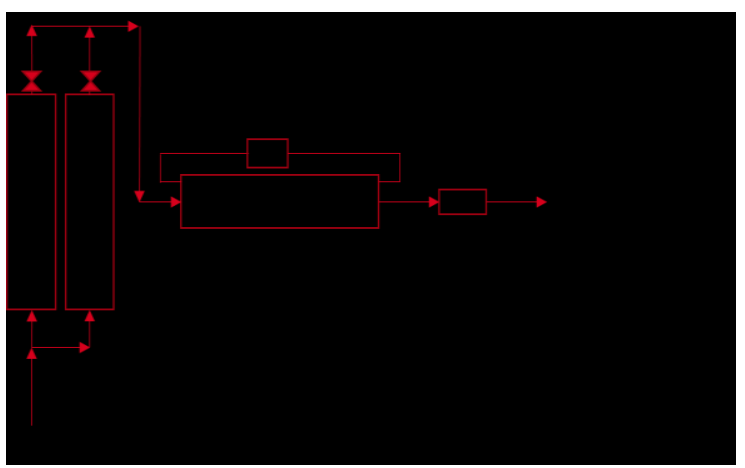

Figure 1: Simple schematic of multiphase core flood apparatus with the densitometer (DMA) located at the outlet of the porous media.

\section{Methodology}

The Anton Paar density meter (DMA HPM) operates on the principle of the oscillating U-tube, which has been used for over 50 years. The U-tube is excited by applying an electrical voltage to a Piezo element, causing it to oscillate at a characteristic frequency [3]. When fluid enters the measurement cell, the oscillation frequency is changed and is accurately detected by a second Piezo element. Each fluid has a characteristic frequency which correlates to its density, as per the formula:

$\rho=\mathrm{A} \tau^{2}-\mathrm{B}$

Where $\mathrm{p}$ is the density of the fluid, A and B are instrument constants, and $\tau$ is the oscillation period. The instrument constants A and B are determined in an instrument adjustment, where two reference standards, such as dry air and pure degassed water, are measured in order to determine the relationship between density and the period of oscillation. Using this relationship, unknown sample densities can be determined using their oscillation. The measuring cell is made from Hastelloy C-276, which can withstand high mechanical stress and is highly chemical resistant. The stated cell volume is $2 \mathrm{~mL}$; however, the sensitive measuring volume is $0.35 \pm 0.03 \mathrm{~mL}$ [2].

Multi-phase core floods were performed using a densitometer placed at the outlet of the sand pack. Density was measured at a frequency of 1 hertz and all measurements were made at the same temperature and pressure as the sand pack by using a circulating water bath and a back-pressure regulator (BPR). The density meter was placed so that all fluid entering the sand pack passed through the measuring cell, before reaching the BPR and fraction collector. Flow rates used were $<0.35 \mathrm{ml} / \mathrm{sec}$, in order to ensure that all fluid passing through the measuring cell was measured. If the flow rate exceeds the limit of the sensitive measuring volume, the accuracy of the analysis is affected.

Steam floods were performed in such a way that additives could be mixed with superheated steam resulting in wet steam laden with chemical additives that are present in the liquid phase of the injected mixture. These mixtures produced an increase in pressure drop relative to the back pressure of the apparatus which was then normalized against the pressure drops of steam of similar total heat values. Table 1 and Table 2 show the details of steam/steam foam experiments.

The dynamic saturation test was performed with nitrogen and water co-injected at a 4:1 gas to liquid ratio at varied flow rates to evaluate the equilibrium water saturation for a given flow rate at high-pressure high-temperature conditions. The back pressure of the 
system is adjusted in such a way that compressibility of flow is negligible and therefore unaccounted for.

Table 1: The sand pack dimensions, geometry, permeability, porosity, composition, and pressure tap locations for steam foam experiments.

\begin{tabular}{|l|l|}
\hline Dimensions & $40.84 \mathrm{~cm} \mathrm{X} 2.244 \mathrm{~cm}$ \\
\hline Geometry & Cylindrical Unconsolidated \\
\hline Permeability & 2.27 Darcy \\
\hline Porosity & $40.14 \%$ \\
\hline $\begin{array}{l}\text { Composition of } \\
\text { Media }\end{array}$ & Silica Sand $140-270$ \\
\hline Tap Locations & $\begin{array}{l}\text { Absolute Pressure at } 0,10 \mathrm{~cm}, \mathrm{dP} \\
\text { at } 10,20,30,40 \mathrm{~cm}\end{array}$ \\
\hline $\begin{array}{l}\text { Operating } \\
\text { Pressure }\end{array}$ & $200 \mathrm{psig}$ \\
\hline $\begin{array}{l}\text { Operating } \\
\text { Temperature }\end{array}$ & $200{ }^{\circ} \mathrm{C}$ \\
\hline
\end{tabular}

Table 2: The sand pack dimensions, geometry, permeability, porosity, composition, and pressure tap locations for the dynamic fluid saturation experiment.

\begin{tabular}{|l|l|}
\hline Dimensions & $30.65 \mathrm{~cm} \mathrm{X} 1.5748 \mathrm{~cm}$ \\
\hline Geometry & Cylindrical Unconsolidated \\
\hline Permeability & 35.52 Darcy \\
\hline Porosity & $35.87 \%$ \\
\hline Composition of Media & Silica Sand $50-70$ \\
\hline Tap Locations & Inlet, Outlet \\
\hline Operating Pressure & $750 \mathrm{psig}$ \\
\hline Operating Temperature & $100^{\circ} \mathrm{C}$ \\
\hline
\end{tabular}

\section{Example Calculations}

Olsen et al. (2017) determined a method for using inline densitometry for quantification of produced water and oil in a two-phase system using the following equation [2]:
CumProdWater $(0, N)=$

$\sum \frac{N}{0} \frac{\text { MeasDens }(i)-\text { LowerEnvelope }(i)}{\text { Upper Envelope }(i) \text {-LowerEnvelope }(i)} *[\operatorname{InjVol}(i)-$ InjVol $(i-1)]$

Where the Lower Envelope is the density of the lightest phase, and the Upper Envelope is the density of the heaviest phase, oil and water, respectively.

The produced oil fraction can then be determined by subtracting the produced water from the total injection volume

$\operatorname{CumPr}(0, N)=\operatorname{CumInjFluid}(0, N)-\operatorname{CumProdWater}(0, N)$

It is noted that the upper and lower envelopes must be carefully determined in order to ensure the accuracy of the analysis.

This equation can also be applied to miscible displacements, where the densities of the two pure phases are known.

In the case of highly viscous oil, the density measurement is dampened by a coating of oil on the walls of the measurement cell. This, in turn, lowers the apparent density. In this case, a moving upper envelope must be used. This is determined by applying a macro to the data set, which detects the change in the upper-density value:

Moving Upper Envelope

$=I F\left(\frac{\text { Measured Density }(i)-\text { Measured Density }(i-1)}{\text { CumProdVol }(i)-\text { CumProdVol }(i-1)}\right)$ (3)

$>0.001$, Upper Envelope $(i-1)$, Measured Density Value

Liquid volumetric fraction calculation:

$$
L V F=\frac{\rho \text { Measured }-\rho G a s}{\rho \text { Water }-\rho G a s}(4)
$$

Therefore, the vapor volumetric fraction can be assumed to be

$V V F=1-L V F$

The produced water/liquid can be calculated when the system pressure is significantly higher than the phase envelope pressure (boiling pressure) of the liquid phase. This is executed in discrete steps with each interval being each individual density measurement.

\section{Produced Liquid per Data Interval $=L V F *$ $\frac{\text { Total Volume Produced }}{\text { Unit Time Unit Time }}$

Using the above equation, the cumulative produced liquid can be calculated as follows: 
Cumulative Produced Liquid $=$

$\sum_{0}^{N}$ Produced liquid per Data Interval

Then using that information we can calculate the liquid saturation within the porous media as follows: $S_{w}=$

Cumulative Produced Liquid-Cumluative Injected Liquid Pore Volume

For steam vapor, volumetric qualities are calculated in the same way as Equation (4)

The mobility reduction of steam foams is expressed as a mobility reduction factor.

Residual Resistance Factor $(R R F)=$ Pressure Drop of Steam with Additive Equiblibirum Pressure Drop of Saturated Steam

At steady state the equation for volumetric flow rate

Volumetric Flow Rate $=\frac{\text { Mass }}{\text { Unit Time }} \times \frac{\text { Unit Volume }}{\text { Mass }}(10)$

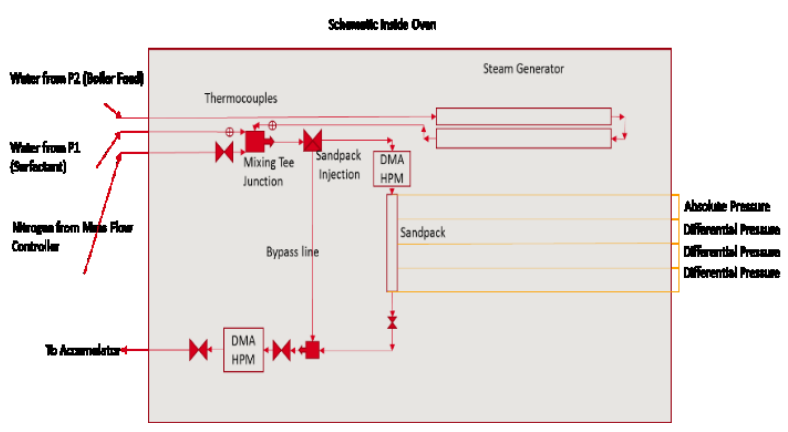

Figure 2: Schematic of the oven internals during steam flood/ hot gas flood experiments, the hot gas flood and the $170{ }^{\circ} \mathrm{C}$ steam flood only had one differential pressure measurement. The density measurement cell was inside the oven at 0.494 cubic centimeters of dead volume downstream the outlet of the porous media for all high-temperature experiments discussed in this paper.

\section{RESULTS AND DISCUSSIONS}

Here we summarized the capability of a highresolution inline densitometer for flow experiments in porous media considering various conditions including high temperature, high pressure, variable oil viscosity, and density differentials.

\section{OIL RECOVERY MONITORING AND BREAKTHROUGH TIME}

The objective of this section is to show how we can precisely monitor the fluid production namely oil recovery in dynamic flow experiments.

The densitometer can be used to accurately determine initial oil in place (OOIP). Additionally, mass balance errors associated with the effluent collection and analysis techniques are eliminated by providing in-line results. Figure 3 to Figure 5 show the densitometer results for conventional displacing experiments with Dodecane as the oil phase.

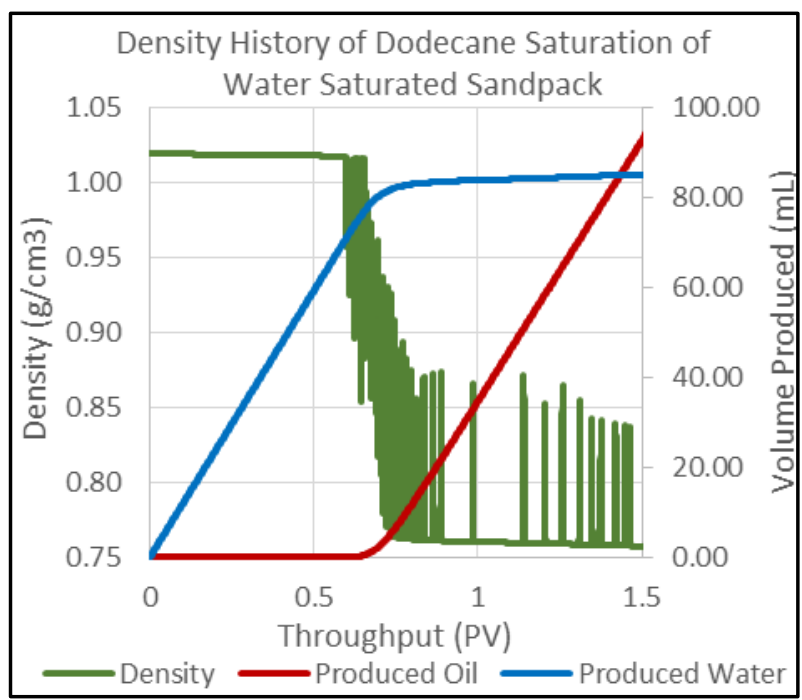

Figure 3: Oil displacing water in water-saturated sand pack. Density monitoring for determination of original oil in place.

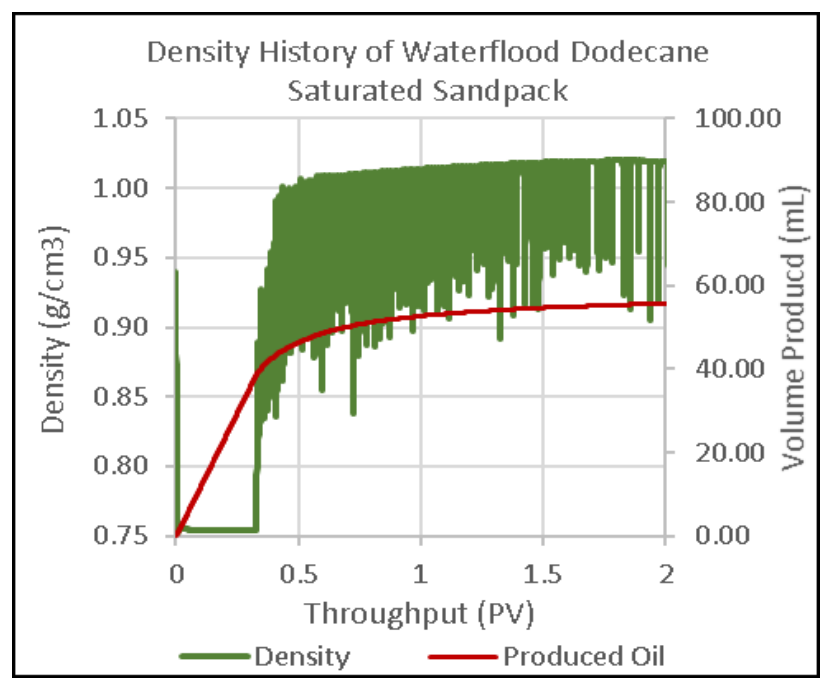

Figure 4: Density history of waterflood through Dodecane saturated sand pack.

As shown in Figure 4, the high-resolution monitoring of effluent from a flow experiment through porous media in a system with two phases of known densities enable oil production to be accurately quantified. The frequency of measurements results in a highresolution history of breakthrough times and fluid behavior. 


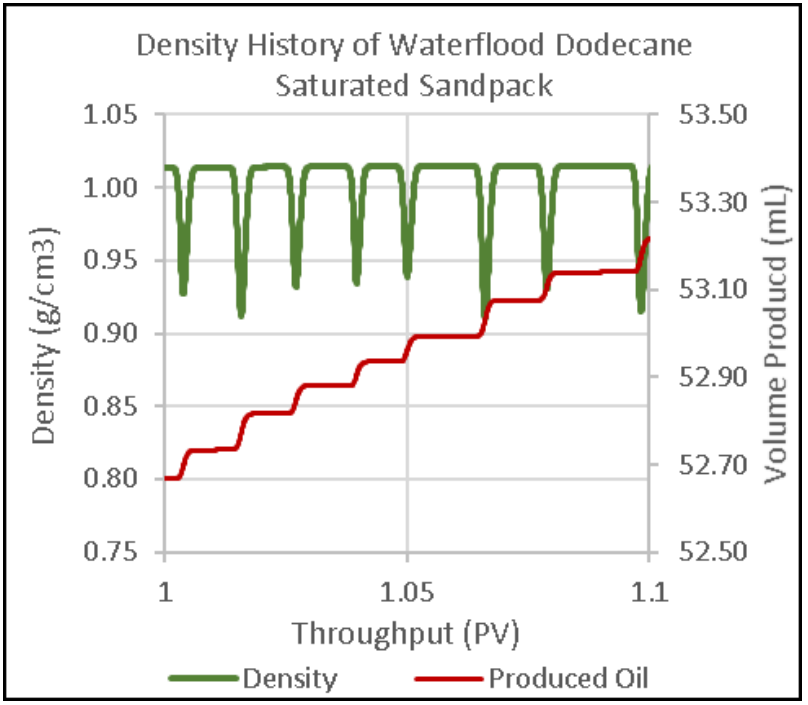

Figure 5: A closer look at density history of waterflood through Dodecane saturated sand pack

A closer look at the density history enables us to identify that the oil is being produced in "trains" (Figure 5) rather than as an emulsion, which is similar to the observation made by Olsen et al. [2]. This is because the density levels off at the density of the water phase, based on the calibration, before dipping down towards the density of the oil. If the oil was being produced as an emulsion, there would be smaller oscillations in density, at a density representative of the quality of the emulsion.

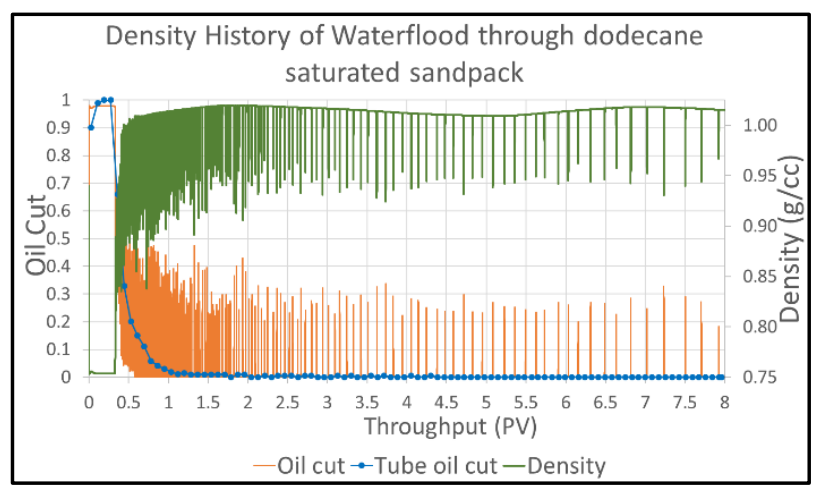

Figure 6: Comparison of density calculation to the manual determination of oil production.

Here we demonstrate that the densitometer provides reliable results when compared to effluent fraction collection and analysis. The mass balance from the effluent analysis was $99.6 \%$ and the mass balance from calculated throughput analysis of the densitometer was $100.2 \%$. The mass balance can be improved by utilizing a circulating water bath in the measurement cell of the densitometer, which was considered in the next section. As shown in Figure 6, effluent analysis cannot accurately capture the oil production performance, especially after $2 \mathrm{PV}$. On the other hand, the oil cut from density calculation shows that the oil is still producing even after $8 \mathrm{PV}$ of the injection. The change in amplitude of the density curve provides us information about the volume of oil production. The change is the frequency of the density will tell us how often the oil is producing. For example, in Figure 6, the frequency of density after 5 $\mathrm{PV}$ is lower than that of the early time stage (1-2 PV) supporting the fact that the oil production rate decreases after $5 \mathrm{PV}$.

\section{Heavy oil recovery calculation; potential and challenges}

As shown above, using the density meter for oil saturation allows for a quick determination of original oil in place. This is more crucial when working with heavy crude oil. The effluent analysis is difficult with heavy oil, as it coats the collection vessel and makes visual determination inaccurate. Solvent separations are also time-consuming.

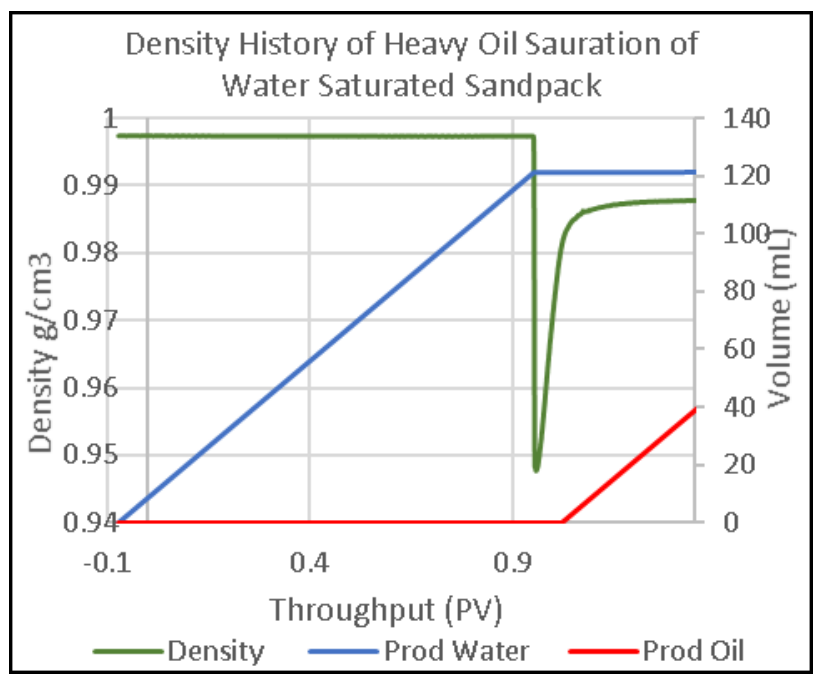

Figure 7: Density history of heavy oil saturation in water saturated sand pack showing breakthrough time.

To verify the effectiveness of oil saturation with the density meter the effluent was passed through the measurement cell and collected into a separatory funnel. The OOIP was $111 \mathrm{~mL}$ from analysis of the separatory funnel and $113 \mathrm{~mL}$ by the densitometer. The percent relative difference between the two methods was $1.79 \%$. 


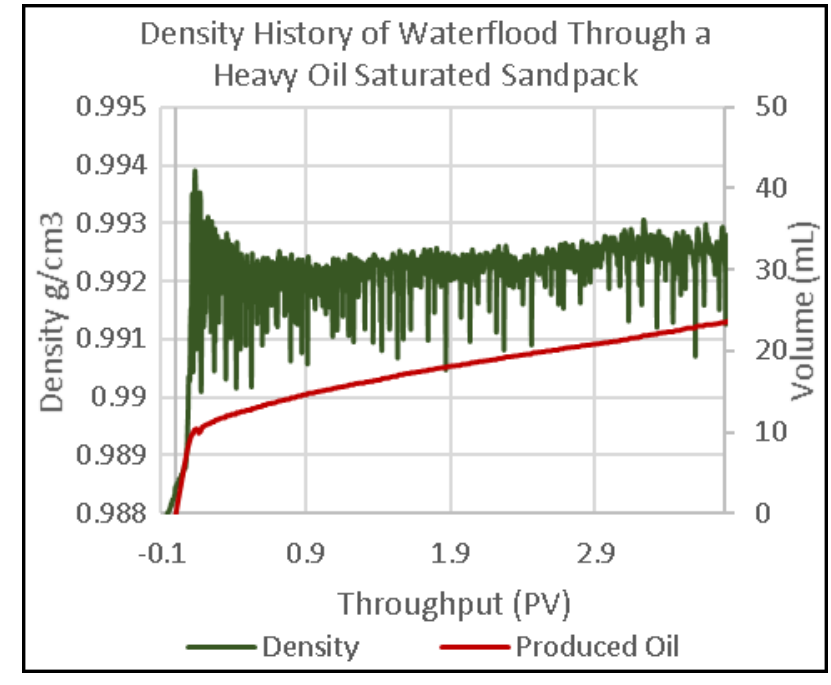

Figure 8: Density history of waterflood through heavy oil saturated sand pack

The quantification of heavy oil through the densitometer is challenging, due to its tendency to coat the walls of the measurement cell. However, if the upper envelope (UE) is adjusted accordingly (See equation 3- Moving upper envelope), it is possible to obtain accurate quantitation of heavy oil production. In order to verify the densitometer results, the effluent was collected into a separatory funnel and analyzed. According to the manual method, $23.6 \mathrm{~mL}$ of oil was produced. According to the density meter results, 24.8 $\mathrm{mL}$ of oil was produced. The methods had a percent relative difference of $5.1 \%$. It should be mentioned that several core flood tests with heavy oil were performed and the average percent relative difference was less than $10 \%$. The density meter eliminates challenging effluent analysis, including the need for time-consuming Dean-Stark analysis.

\section{Nanoparticle transport in porous media}

There is a linear relationship between the density and concentration of nanofluid, enabling the densitometer to be used as an inline analyzer for quantification of nanofluid in a two-phase system (Figure 9). Errors could arise from adsorption of a stabilizing agent to the porous media and fines migration that can be potentially detected by high-resolution density measurement.

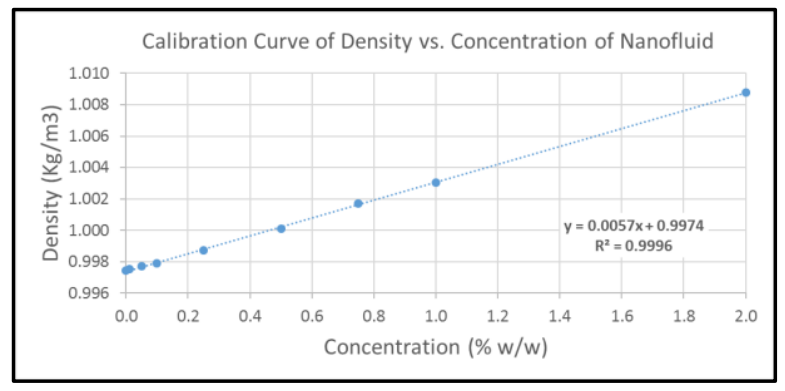

Figure 9: The density of silica nanoparticle at different concentrations. High-resolution density measurement allows accurate detection of nanoparticle with a relatively small increment of nanoparticle concentration.

Following is an example of densitometer capability to detect nanoparticle transport in porous media. The results were also correlated with the magnetic susceptibility measurement of the effluent samples. A suspension of iron oxide nanoparticles was injected in a water-saturated porous media (properties of porous media is mentioned in Table 2) at room condition. Thereafter, the nanofluid displaced with post flush of water. The iron oxide nanoparticles have magnetic properties so they can be detected by magnetic susceptibility measurement. The magnetic susceptibility is normalized based on the magnetic susceptibility of porous media without iron oxide nanoparticles (Figure 10b) any value above zero shows the presence of iron oxide nanoparticles. More detail on such experiments and magnetic susceptibility measurement can be found elsewhere [4].

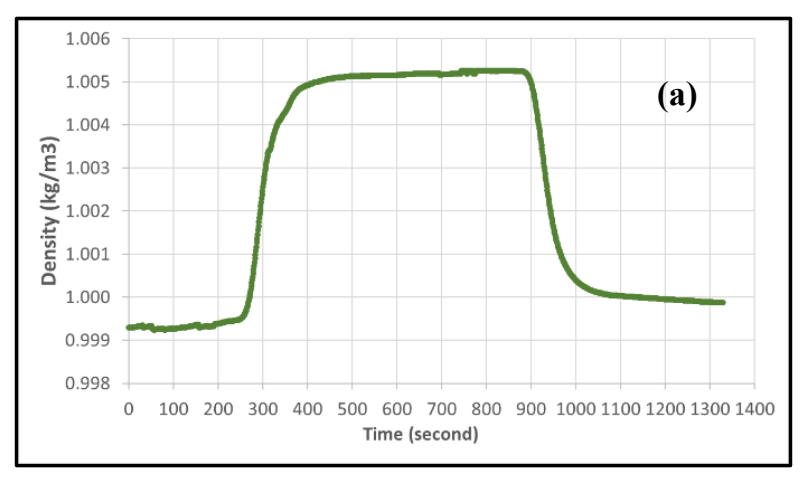

The normalized magnetic susceptibility profile shows that the particle breakthrough occurred around 250300 minutes. However, the density profile can accurately show the breakthrough time (260 seconds) since it is a continuous measurement with $1 \mathrm{~Hz}$ frequency. 


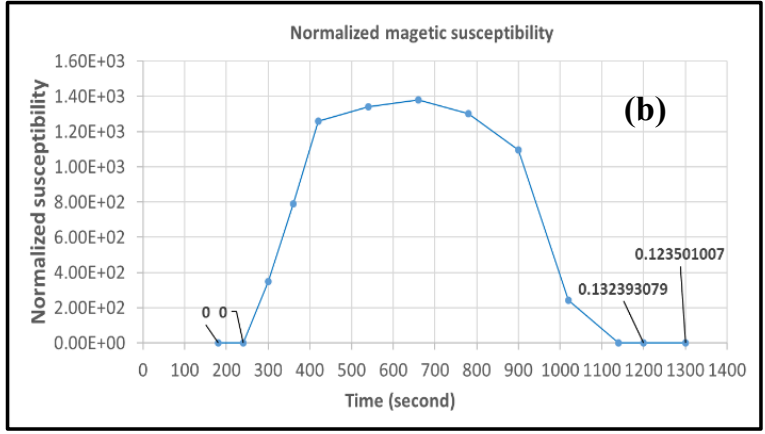

Figure 10: Density profile (a) and magnetic susceptibility profile (b) of iron oxide nanoparticle showing the breakthrough time and nanoparticle transport behavior. Post flush of water started at 600 seconds.

Interestingly the density profile at $1200-1300$ seconds is higher than the beginning (water density) suggesting the nanoparticles still present in the effluent sample. The dispersed iron oxide nanoparticles in water create a very dark color liquid which is easily detectable with the naked eye even in low concentration (e.g. $0.05 \mathrm{wt}$ $\%$ ). The effluent sample at 1200 and 1300 seconds in Figure $10 \mathrm{~b}$ was clear by naked eyes, but the susceptibility measurement suggests that there is still nanoparticle in porous media supporting the density results.

\section{Monitoring saturated steam and hot gas multiphase flows through porous media}

Steam/saturated steam flow in porous media presents an especially complex multiphase flow scenario where densitometry can provide some qualitative and semiquantitative data. High-resolution densitometry enables us to monitor -but not quantify- steam condensate production of the porous media, and we observe distinct variances during foam production as it compares to wet steam flow. Volumetric steam qualities can also be calculated from the density histories, and consequently, mass qualities are also able to be calculated when assuming the system is at steady state.

Steam flood (baseline) is from 4000 seconds to 6850 seconds as shown in Figure 11. This flood had a flash calculated steam quality of injection of 0.4-0.6, however, the flash calculation does not account for the heat losses associated with the steam moving through the stainless-steel tubing while being injected into the porous media. Densitometry enables the analyst to have experimental data to determine what the true steam qualities of injections are. In this scenario, the mass flow rate of the steam was $10 \mathrm{~g} / \mathrm{min}$ and the thermodynamic flash calculations provide a mass quality result of $50 \%+/ 10 \%$ while the density meter results are less than $10 \%$ mass quality for both the inlet and the outlet. The density meters placed at the inlet and outlet also enables us to observe that in this experiment, the outlet mass quality is higher than at the inlet due to flashing off liquid as the absolute pressure decreases during propagation through the porous media.
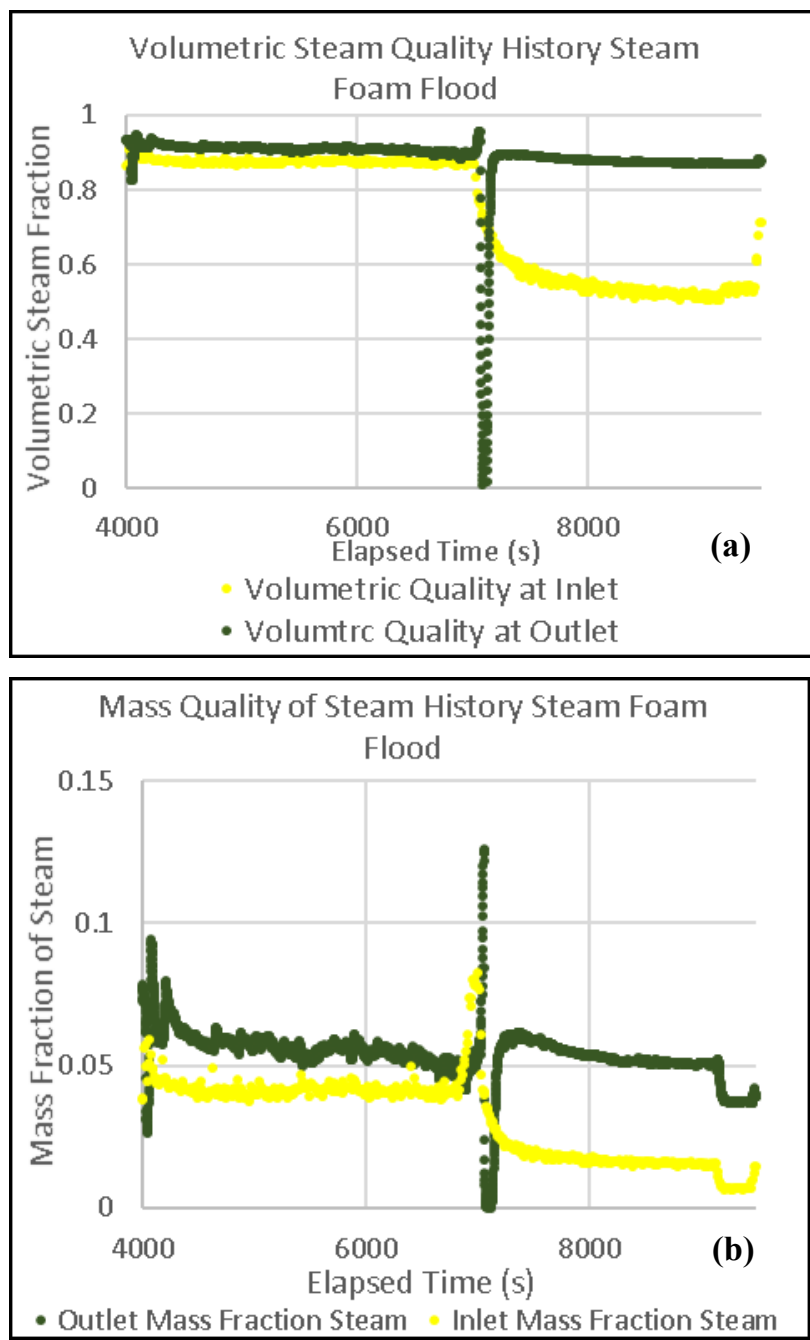

Figures 11: Monitoring volumetric (a) and mass (b) steam quality (inlet/outlet) with the help of inline densitometer. An ambient oven temperature of $170{ }^{\circ} \mathrm{C}$ was used. 


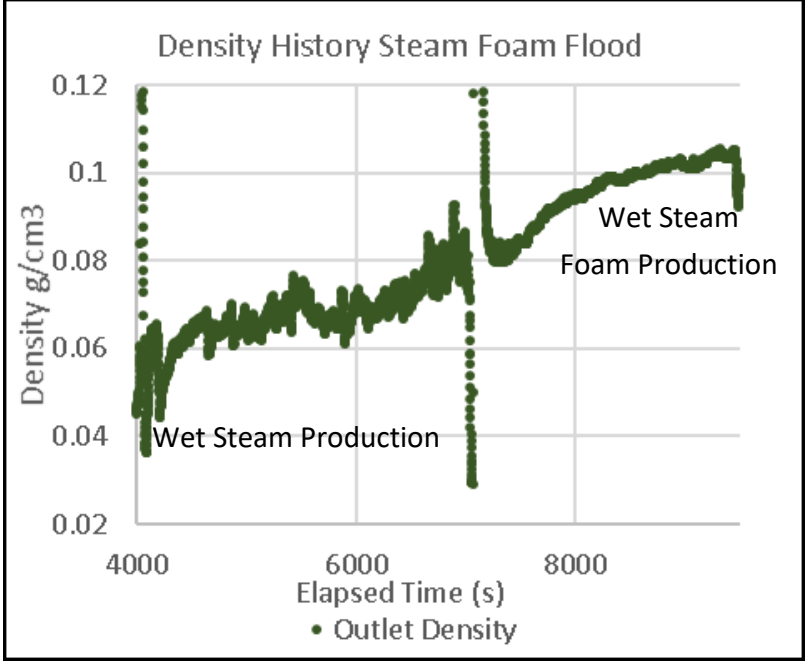

Figure 12: Foam/no-foam boundary identification for steam foams at the outlet of porous media. An ambient oven temperature of $170{ }^{\circ} \mathrm{C}$ was used.

Figure 12 shows the steam (4000 seconds to 6850 seconds) and steam foam flood experiment. The steam foam flow has a distinctive characteristic compared to the pure steam flow at the same steam quality. Steam foam production has a distinct lack of random fluctuations that are observed in the saturated steam production.

Steam condensation can be monitored with the density profile (Figure 13). This will enable us to evaluate the breakthrough times of steam and find out when vapor began being produced at the outlet.

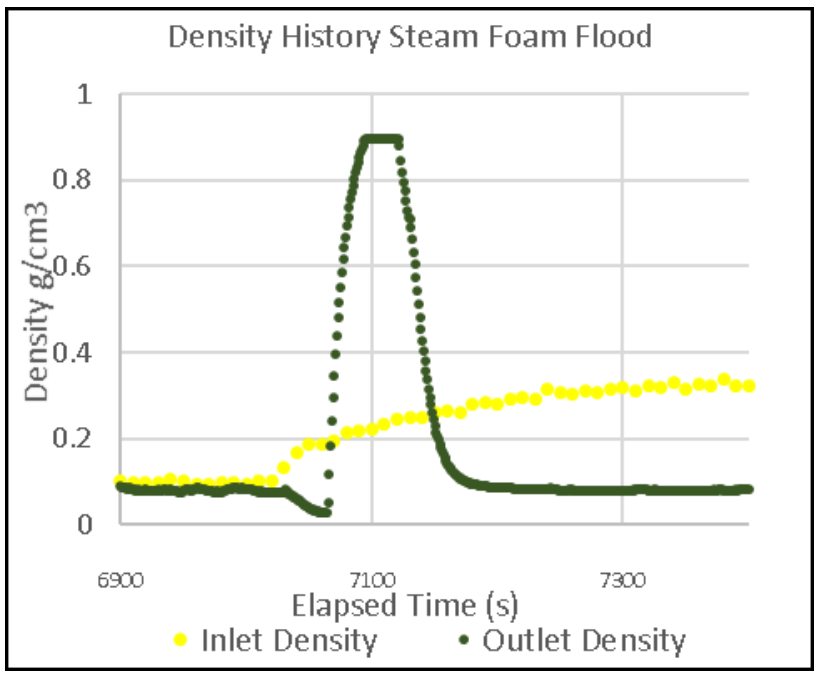

Figure 13: Steam condensation monitoring with the help of inline densitometer.

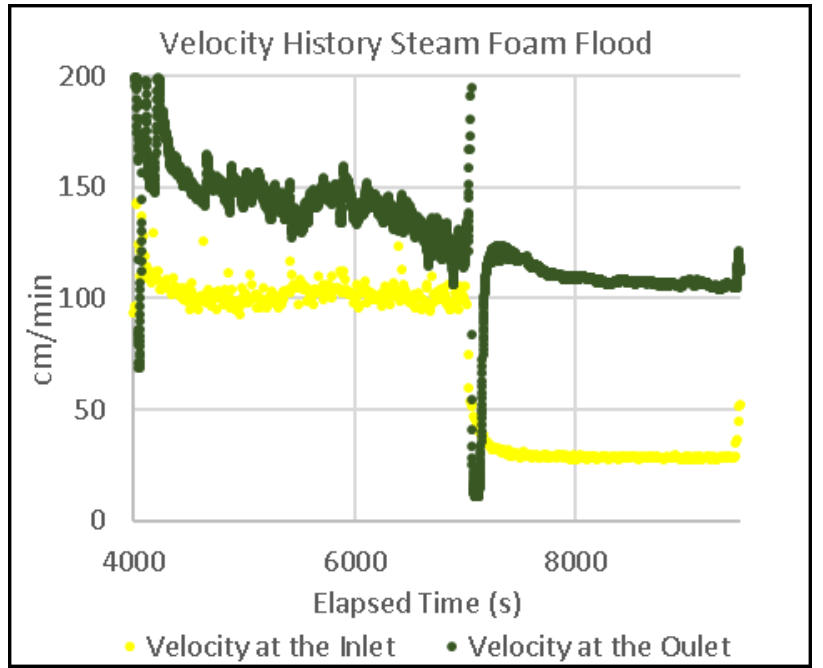

Figure 14: The true velocities of steam moving into the porous media. Steam flood (baseline) is from 4000 seconds to 6850 seconds. An ambient oven temperature of $170{ }^{\circ} \mathrm{C}$ was used.

The true velocities of steam cannot accurately be calculated with flash calculations and heat loss modeling. Figure 14 demonstrates the capability of calculating volumetric flow rates during steam flooding. This is not possible to calculate accurately without the use of densitometry due to the complex nature of the heat transfer that is involved with steam floods such as oven convention speeds, insulation, etc. Calculating the velocity based on flash calculations results in a falsely high velocity. The calculations all assume the system in is a steady state. 


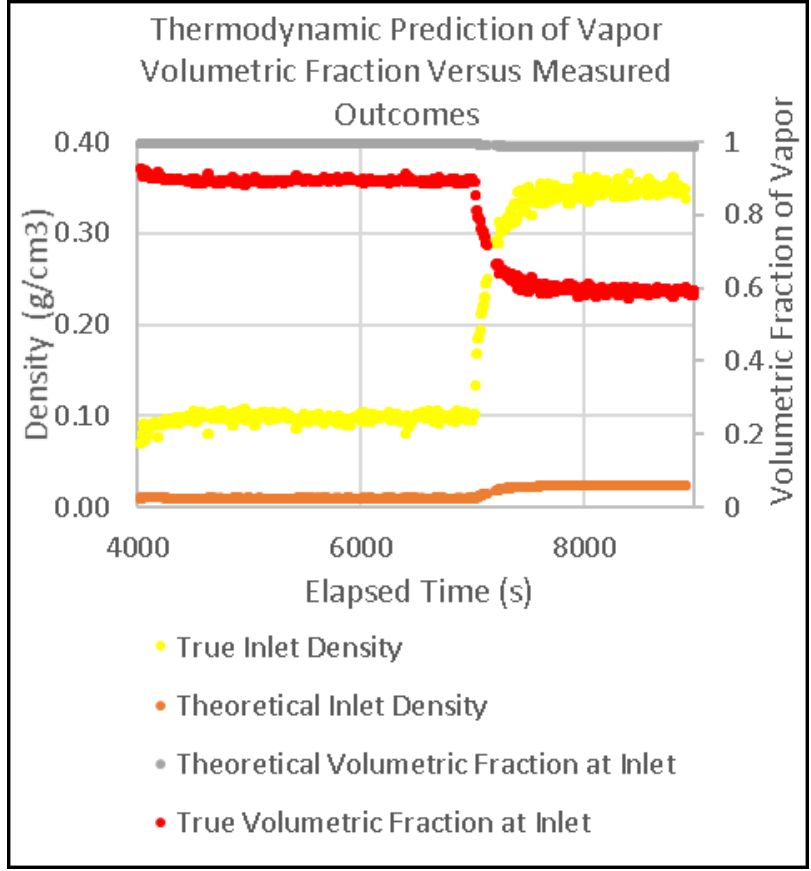

Figure 15: A comparison of the theoretical calculated density and the volumetric fraction of the steam being injected into the porous media, highlighting the comparison between thermodynamic estimations of steam volumetric quality and measured steam volumetric quality using the density meter.

The true velocities of the being injected into the porous media can readily be calculated using the stream density and the mass flow rate which provides us with accurate flow velocities of steam. Using the density meter has enabled more accurate predictions of velocities than with flash calculations alone due to real-time measurements of density, or specific volume of the injected phase as highlighted in Figure 15. The instrument also provides a without a doubt assurance of what phase of the state the injected fluid is in which aides greatly in steam foam floods since occasionally rapid changes in pressure cause condensate production. In some cases, it's helpful to troubleshoot whether the performance of a chemical is simply due to the lack of the presence of a steam phase (instrument limitation) or to distinguish that a chemical simply does not perform in the presence of the condensable gas phase.

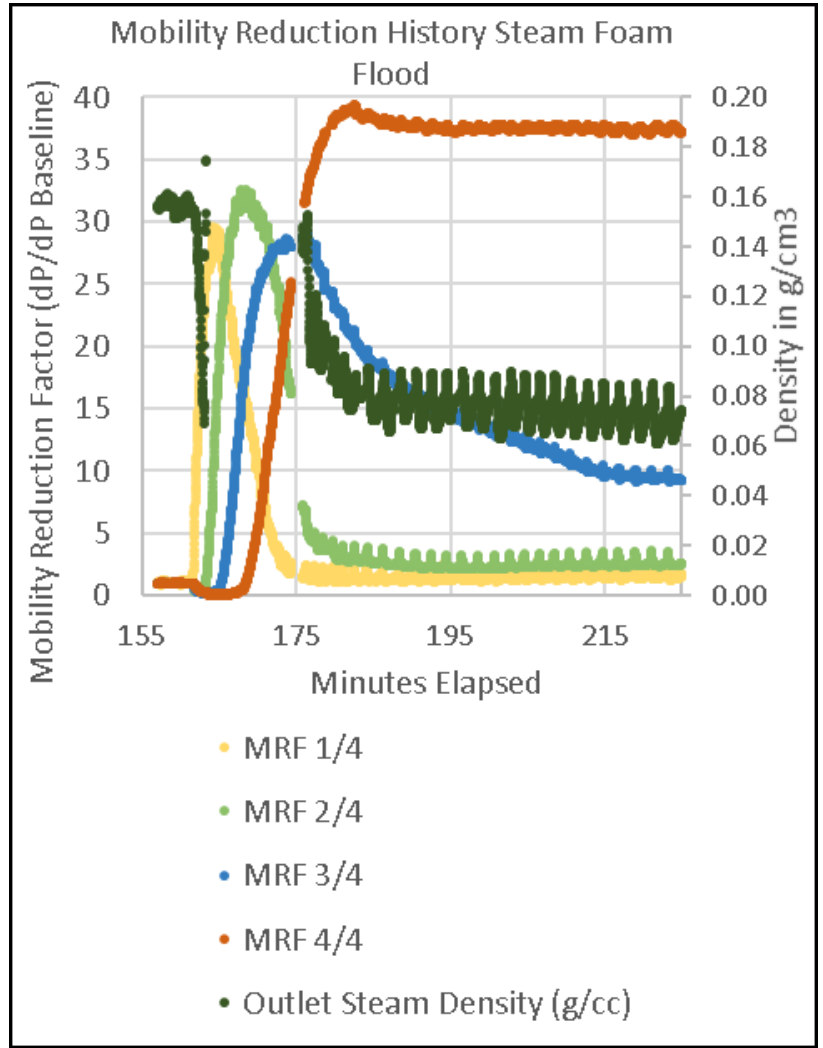

Figure 16: Mobility reduction factor and density profile during steam foam injection. The baseline was performed from 130 minutes until 165 minutes, which is only partially visible in this plot. This steam flood was performed at 200 ${ }^{\circ} \mathrm{C}$ with the outlet at the saturation pressure regulated with a back-pressure regulator.

As shown in Figure 16, initially we see the equilibrium steam density at baseline. The mixing water is then forced to stop mixing with the superheated steam prior to injection and is substituted by a specially designed foaming solution. Immediately a reduction in density occurs simultaneously to the increase of differential pressure in the first quarter of the sand pack. As resistance to flow increases the bubble point temperature of the steam also increases, this means that heat must be delivered to the porous media in the form of latent heat which generates condensate. We also observe the true breakthrough of the steam phase, which normally would be assumed to be at the maximum differential pressure of the last section of the sand pack, however, we can observe that steam actually begins to forms at the maximum differential of the $3^{\text {rd }}$ quarter of the sand pack. This is caused by the flashing of the condensate and should not be considered the actual breakthrough time of the injected steam front. 


\section{Hot gas injection flow monitoring}

The ability to dynamically measure liquid saturation allows us to analyze the equilibrium water saturations during multiphase flow conditions as shown in Figure 17.

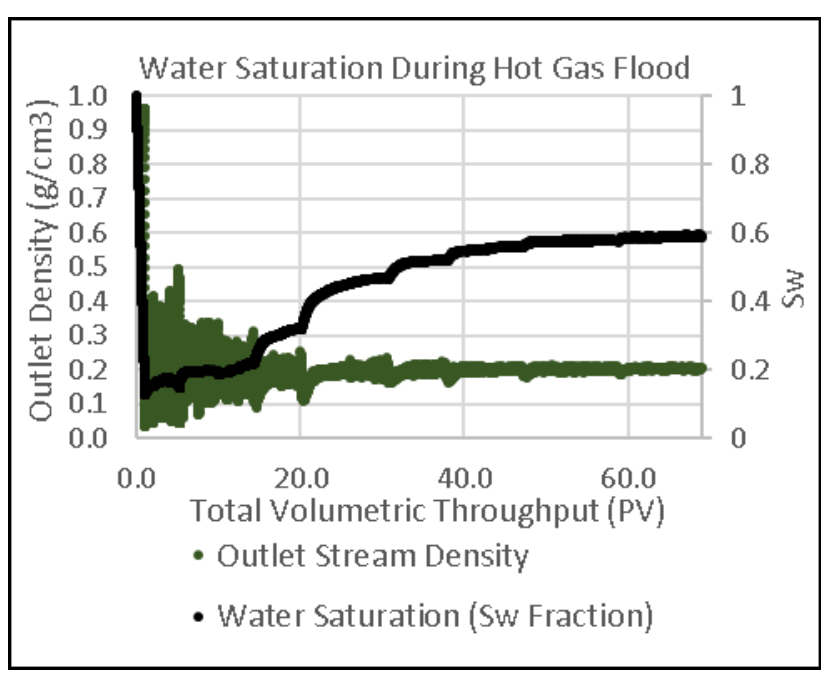

Figure 17: A plot demonstrating the use of densitometry to calculate dynamic fluid saturation. This test was performed at $100{ }^{\circ} \mathrm{C}$ and $750 \mathrm{psig}$ back pressure. Total Volumetric flow rates were $0.5,1.0,1.5,3.0,6.0,10.0,15.0,20.028 .0$ $\mathrm{mL} / \mathrm{min}$ respectively

\section{Quality Control of Dynamic Fluid Saturation}

It should be noted that beyond $\sim 21 \mathrm{cc} / \mathrm{min}$ does not provide the density meter with enough time to measure "every molecule" that passes through it, the residence time within the measurement cell is not long enough however the saturation values still aligned quite closely with manual mass balance performed by weighing the isolated sand pack before and after the flood. Manual mass balance results in a water saturation of 0.497 in comparison to the 0.587 . The relative difference between the two methods is $16.6 \%$. According to manual mass balance, $10.66 \mathrm{~mL}$ of water was left within the sand pack, and according to the densitometer $12.56 \mathrm{~mL}$ of water are left inside the sand pack, this error could be due to the fact that once velocities above $\sim 21 \mathrm{cc} / \mathrm{min}$ were used at the last two velocity intervals the materials flowing through the densitometer did not have enough residence time to be accounted for in the cell. The error is magnified in the $\mathrm{S}_{\mathrm{w}}$ calculations because of the reduced pore volume, a larger pore volume would reduce the impact of these uncertainties. The total volume (of water) injected was $281.67 \mathrm{cc}$, the total volume produced was $281.08 \mathrm{cc}$. A mass balance deficit of $-0.2 \%$ is obtained. The densitometer has proven to be an effective tool for mass for two-phase flow based on the data obtained.

\section{Summary}

Understanding fluid flow in porous media is challenging. Laboratory experiments at real reservoir condition are key to achieve this goal. However, the nature of experiments (pressure, temperature, fluid viscosity, multiphase flow, etc.) makes the data analysis and interpretation challenging and sometimes impossible. This study introduces the high-resolution inline density measurement at experimental condition (i.e. high pressure, high temperature) as a unique tool for understanding fluid flow in porous media. Following are some of the unique achievement of data analysis utilizing densitometer (DM):

- Besides oil recovery calculation in conventional light oil experiments, DM can significantly reduce the time, chemical usage for oil recovery calculation in tests including heavy viscous oil without compromising the accuracy.

- Valuable information in hightemperature experiments including monitoring steam condensation, steam/steam foam propagation and breakthrough time, water saturation changes, and steam front velocity calculation.

- Understanding complex fluids flow by identifying foam/no foam boundary at reservoir condition without the need for visualization.

- Monitoring and detecting nanoparticles or fine transport in porous media.

\section{References}

[1] A. Baldygin, D. S. Nobes, and S.K. Mitra. New Laboratory Core Flooding Experimental System. Industrial \& Engineering Chemistry Research 201453 (34), 13497-13505 (2014).

[2] D. Olsen. Using a Densitometer for Quantitative Determinations of Fluid Density and Fluid Volume in Core Flooding Experiments at Reservoir Conditions. The International Symposium of the Society of Core Analysts, Vienna, Austria, 27 August - 1 September (2017). 
[3] Instruction Manual, DMA HPM, Document no. C34IB06A.fm, Anton Paar GmbH, Graz, Austria (2005).

[4] A., Donath. Magnetic Imaging of Nanoparticles Flow through Porous Media. Master thesis, University of Calgary (2017). 